\title{
Identification of diterpenoid compounds that interfere with Fli-1 DNA binding to suppress leukemogenesis
}

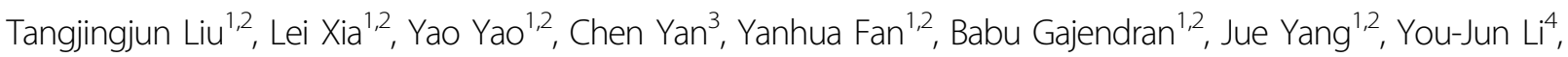 \\ Juan Chen ${ }^{4,5}$, Jorge Filmus ${ }^{5}$, David E Spaner ${ }^{5}$, Eldad Zacksenhaus ${ }^{6,7}$, Xiaojiang Hao ${ }^{1,2,3}$ and Yaacov Ben-David ${ }^{1,2}$
}

\begin{abstract}
The ETS transcription factor Fli-1 controls the expression of genes involved in hematopoiesis including cell proliferation, survival, and differentiation. Dysregulation of Fli-1 induces hematopoietic and solid tumors, rendering it an important target for therapeutic intervention. Through high content screens of a library of chemicals isolated from medicinal plants in China for inhibitors of a Fli-1 transcriptional reporter cells, we hereby report the identification of diterpenoid-like compounds that strongly inhibit Fli-1 transcriptional activity. These agents suppressed the growth of erythroleukemic cells by inducing apoptosis and differentiation. They also inhibited survival and proliferation of B-cell leukemic cell lines as well as primary B-cell lymphocytic leukemia (B-CLL) isolated from 7 patients. Moreover, these inhibitors blocked leukemogenesis in a mouse model of erythroleukemia, in which Fli-1 is the driver of tumor initiation. Computational docking analysis revealed that the diterpenoid-like compounds bind with high affinity to nucleotide residues in a pocket near the major groove within the DNA-binding sites of Fli-1. Functional inhibition of Fli-1 by these compounds triggered its further downregulation through miR-145, whose promoter is normally repressed by Fli-1. These results uncover the importance of Fli-1 in leukemogenesis, a Fli-1-miR145 autoregulatory loop and new anti-Fli-1 diterpenoid agents for the treatment of diverse hematological malignancies overexpressing this transcription factor.
\end{abstract}

\section{Introduction}

Leukemogenesis involves alterations in multiple oncogenes and tumor suppressor genes as well as disruption of tumor microenvironment ${ }^{1,2}$. Standard therapy including surgery, chemo-, radio- and even targeted-therapy are unsuccessful in curing leukemia. Thus, more potent modalities and patient-tailored therapies are needed to eradicate malignant forms of this disease.

\footnotetext{
Correspondence: Xiaojiang Hao (haoxj@mail.kib.ac.cn) or

Yaacov Ben-David (yaacovbendavid@hotmail.com)

'State Key Laboratory for Functions and Applications of Medicinal Plants, Guizhou Medical University, Guiyang 550025, China

${ }^{2}$ The Key Laboratory of Chemistry for Natural Products of Guizhou Province and Chinese Academic of Sciences, Guiyang, Guizhou 550014, China Full list of author information is available at the end of the article. These authors contributed equally: Tangjingjun Liu, Lei Xia Edited by M. Bushell
}

One major driver of leukemogenesis is the ETS transcription factor (TF), Friend leukemia integration 1 (Fli1), originally identified as a site of common proviral integration in F-MuLV-induced erythroleukemias ${ }^{3}$. Activation of Fli-1 was subsequently confirmed to underlie induction of erythroleukemias by this virus ${ }^{4,5}$. Fli-1 was also identified as a site of specific chromosome 11;22 translocations in childhood Ewing's sarcomas ${ }^{6}$. The chimeric EWS/FLI-1 fusion protein generated from this translocation is a potent oncogene $e^{6}$. Fli-1 exerts its effects by controlling the expression of genes involved in proliferation, differentiation, program cell death (apoptosis) and inflammation, all important hallmarks of cancer ${ }^{7,8}$. Fli-1 also promotes angiogenesis, further contributing to tumor progression ${ }^{7}$. Knockdown of Fli-1 in such tumors potently suppress their growth ${ }^{9}$ indicating that tumors driven by Fli-1 are addicted to its continuous expression.

\section{(c) The Author(s) 2019}

(c) (i) Open Access This article is licensed under a Creative Commons Attribution 4.0 International License, which permits use, sharing, adaptation, distribution and reproduction c. in any medium or format, as long as you give appropriate credit to the original author(s) and the source, provide a link to the Creative Commons license, and indicate if changes were made. The images or other third party material in this article are included in the article's Creative Commons license, unless indicated otherwise in a credit line to the material. If material is not included in the article's Creative Commons license and your intended use is not permitted by statutory regulation or exceeds the permitted use, you will need to obtain permission directly from the copyright holder. To view a copy of this license, visit http://creativecommons.org/licenses/by/4.0/. 
These observations point to Fli-1 as an important therapeutic target for the diverse type of malignancies driven by this oncogene ${ }^{7}$.

In the past decade, various methods were used to target DNA- and RNA-binding activities of EWS-Fli-1 for the treatment of Ewing Sarcomas. These efforts led to the identification of several compounds with potent anticancer activity ${ }^{10-14}$, yet none has been implemented in the clinic. There is therefore an urgent need to identify more specific and potent inhibitors of EWS-Fli-1 and/or Fli-1 with clinical utility. Toward this end, we previously performed high throughput screens to identify drugs that specifically target this TF. Several anti-Fli-1 compounds were identified and shown to block leukemic cell proliferation in culture and leukemogenesis in mouse models ${ }^{10}$. However, these compounds target other proteins in addition to Fli-1, and exhibited various side effects. To identify more potent and specific inhibitors, we here report on a Fli-1 inhibitor screen of a library of chemicals isolated from medicinal plants in China. We identified two chemically related diterpenoid-like compounds that suppress Fli-1 transcriptional activity and its downstream targets, leading to inhibition of B cell lymphoma in vitro and erythroleukemia in a preclinical mouse model. The inhibition of Fli-1 by these diterpenoids subsequently triggered post-transcriptional downregulation of Fli-1 protein levels through upregulation of miR-145. Thus, this work identifies novel inhibitory compounds that can be used for the treatment of cancers driven by overexpression of Fli-1.

\section{Results}

Identification of potent Fli-1 inhibitors from a library of compounds isolated from medicinal plants in China

To identify specific anti-Fli-1 compounds with low toxicity for treating tumors overexpressing this TF, we screened a library of 2000 small, highly purified compounds isolated from medicinal plants in China. As a reporter, we used a plasmid, FB-Luc, in which two Fli-1 binding sites were placed upstream of a minimum promoter of the luciferase PGL-4.28 plasmid $^{10}$. HEK293T cells stably expressing Fli-1 and FB-Luc plasmids were established and used for the screen. Several compounds were identified. Among these, A661 and A665 (Fig. 1a), are structurally related to a family of natural diterpenoids ${ }^{15}$. These compounds strongly inhibited luciferase activity in HEK293T cells co-transfected with FB-Luc and MigR1-Fli-1 relative to control MigR1 expression vector in a dose-dependent manner (Fig. 1b, c). The compounds also inhibited luciferase activity following co-transfection of FB-Luc with MigR1-EWS-Fli-1. Suppression was Fli-1 specific; it was low or marginal with a control CMV-Luc reporter plasmid lacking Fli-1 binding sites (Fig. 1d).

\section{Inhibition of Fli-1 by $A 661$ and A665 in erythroleukemic cells induces apoptosis, cell cycle arrest, and erythroid differentiation}

A661 and A665 inhibited growth of cell lines derived from solid tumors such as breast (MDA-MB-231) and prostate (PC3) as well as from leukemic lines (CB7, Daudi, HEL) with IC50 ranging from 0.9-4.3 $\mu \mathrm{M}$ (Fig. 1e). Among these leukemic cell lines, HEL, Daudi, and CB7 express Fli-1 while the rest are either negative or produce negligible levels of this $\mathrm{TF}^{16}$. SiRNA-mediated knockdown of Fli-1 in both HEL and CB7 cells was previously shown to inhibit cell proliferation through induction of apoptosis and erythroid differentiation ${ }^{9}$. Inhibition of proliferation by A661/A665 in Fli-1-negative cells suggests that these compounds target additional proteins required for cell proliferation (see below). However, in tumors that are driven by Fli-1, this oncogene is the major target of these compounds.

Treatment with A661 or A665 downregulated Fli-1 protein expression in HEL cells (Fig. 1f, g) in a dosedependent manner. Fli-1 is thought to autoregulate itself through several ETS binding sites in its promoter ${ }^{17,18}$. In accordance, both compounds significantly increased mRNA levels of $f l i-1$ (Supplemental Fig. 1).

Compounds A661 and A665 induced rapid apoptotic cell death in erythroleukemic HEL and CB7 cells, 24h post-treatment (Supplemental Fig. 2A). Consistent with this, inhibition of Fli-1 resulted in reduced expression of Bcl-2, a known downstream target of this TF (Fig. 1f, g). In addition to apoptosis, A661 and A665 induced cell growth inhibition, which was associated with a significant increase in the percentage of cells in G2 (Supplemental Fig. 2B). There was also some effect on $S$ phase that was variable between cell lines (Supplemental Fig. 2B). Indeed, A661 and A665 induced cyclin-dependent kinase inhibitor $\mathrm{p} 27^{\text {kip1 }}$ protein expression involved in erythroid differentiation, whereas the Fli-1 agonist TPA suppressed p2 $7^{\text {kip } 1}$ expression (Supplemental figure 3A) ${ }^{16}$.

We previously showed that Fli-1 knockdown induces erythroid differentiation ${ }^{9}$. To determine whether A661 and A665 affect the same differentiation program as Fli-1 knockdown, we quantified the expression of a subset of its target genes that promote erythroid maturation ${ }^{10}$. Specifically, Fli-1 negatively regulates transcription of GATA1 and SHIP-1 involved in erythroid differentiation, and positively regulates transcription of glycoprotein 6 (GP6) required for megakaryocyte development ${ }^{17-22}$. Consistent with this, mRNA levels of the erythroid genes GATA1 and SHIP-1 was induced by both A661 and A665 (Supplemental Fig. 3B, C), whereas expression of GP6 was suppressed (Supplemental Fig. 3D). Expression of $\beta$-globin and glycoporin A (GYPA), both markers of erythroid differentiation, was induced by both compounds, but more by A665 (Supplemental Fig. 3E, F). While MAPK/ 

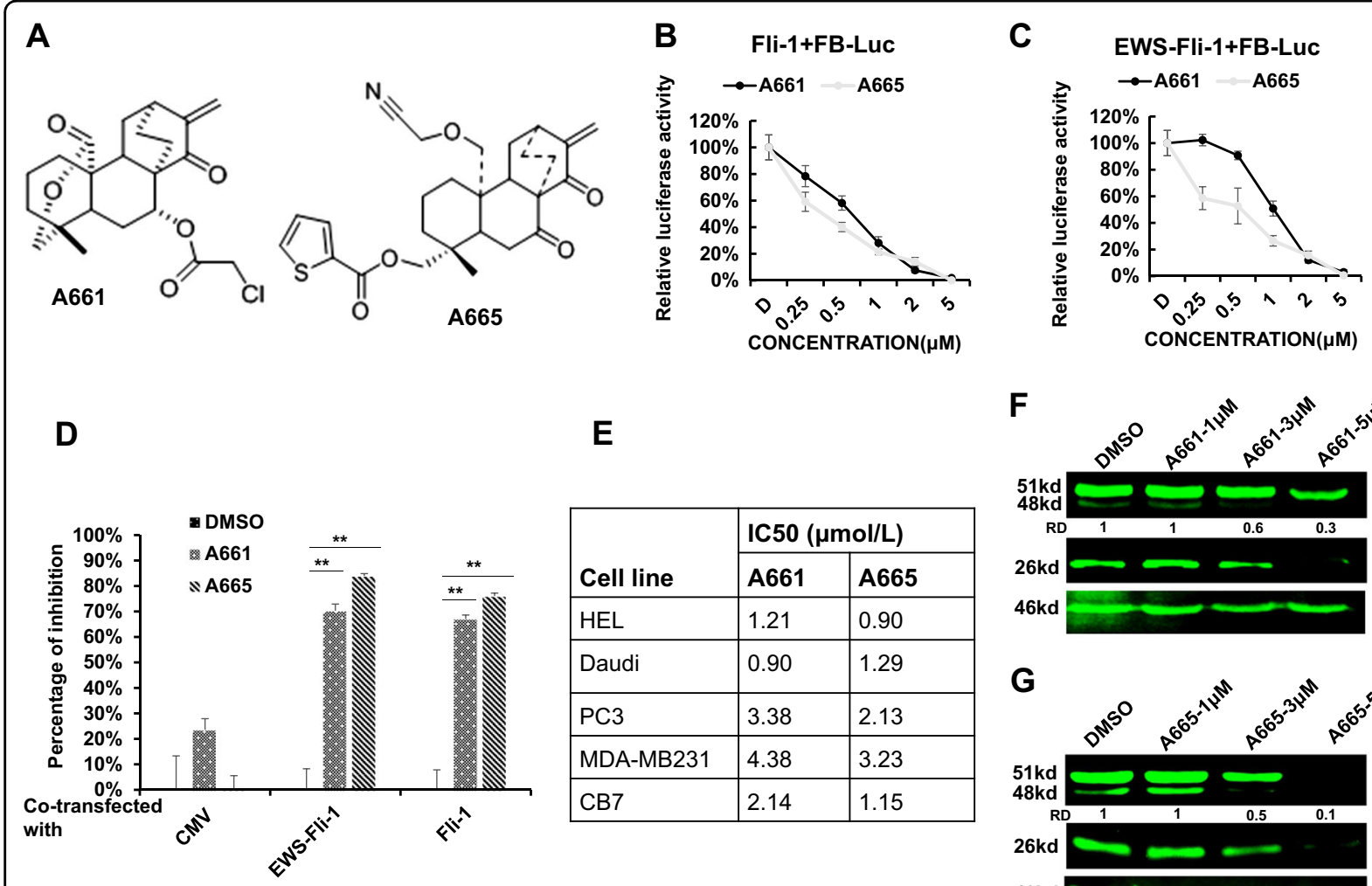

$\mathbf{E}$
\begin{tabular}{|l|l|l|}
\hline \multirow{4}{*}{ Cell line } & AC50 $(\boldsymbol{\mu m o l} / \mathbf{L})$ \\
\cline { 2 - 3 } & $\mathbf{A 6 6 1}$ & $\mathbf{A 6 6 5}$ \\
\hline HEL & 1.21 & 0.90 \\
\hline Daudi & 0.90 & 1.29 \\
\hline PC3 & 3.38 & 2.13 \\
\hline MDA-MB231 & 4.38 & 3.23 \\
\hline CB7 & 2.14 & 1.15 \\
\hline
\end{tabular}
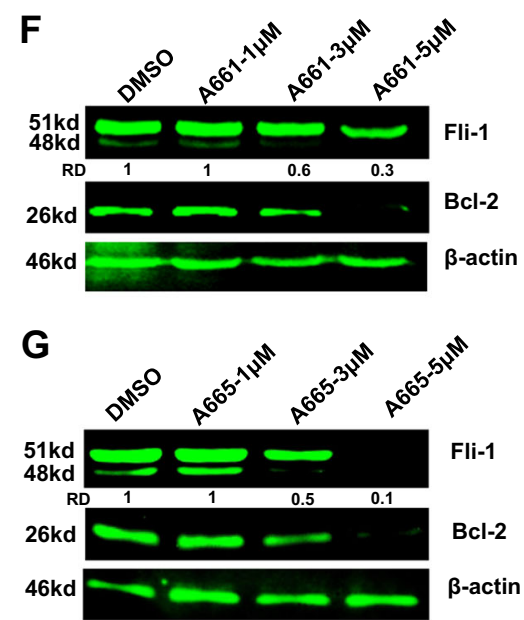

Fig. 1 Diterpenoid compounds A661 and A665 suppress Fli-1 expression. a Chemical structures of the diterpenoid compounds A661 and A665. b, c A665 and A665 suppress transcriptional activity of FB-Luc, co-transfected with Fli-1 (b) or EWS-Fli-1 (c) in a dose-dependent manner. d A661 and A665 $(2.5 \mu \mathrm{M})$ specifically suppress transcriptional activity of FB-Luc, but not control CMV-Luc. e IC50s of A661 and A665 for the indicated cell lines. f, g Western blots for Fli-1 and BCL2 in HEL cells treated with indicated doses of A661 (f) or A665 (g) for $18 \mathrm{~h}$. $\beta$-actin was used as a loading control. * Denotes $P<0.05 ;{ }^{* *}, P<0.005$. RD: relative density

ERK phosphorylation was slightly affected by A661 and A665, expression of c-MYC, an important regulator of erythroid differentiation ${ }^{23,24}$, was significantly downregulated (Supplemental Fig. 4A, B). Overall, these results demonstrate that pharmacological inhibition of Fli-1 by A661 or A665 closely mimics the effect of its genetic ablation by siRNA, indicating that these compounds exert their cellular and molecular effects to a large degree through inhibition of Fli-1.

\section{Computational analysis predicts that $A 661$ and $A 665$ bind near the DNA-binding sites of Fli-1, leading to transcriptional suppression}

Hou et al. ${ }^{25}$ have recently demonstrated strong affinity of mithramycin (MTM), a known inhibitor of EWS-Fli1 , to a GGAA repeat near the minor groove of DNA, which is recognized by this chimeric transcription factor (Fig. 2a, e). This interaction subsequently perturbed binding of the ets domain of EWS-FLI1 to the major groove of this DNA recognition site (Fig. 2e). Through docking analysis, we found that both A661 (Fig. 2b-d) and A665 (Fig. 2f-h) bind within a pocket near minor groove in vicinity of the MTM binding site. This comparative analysis revealed that A661 and A665 have similar binding affinity and docking score with MTM (Fig. 3a). From this analysis, A665 is expected to be a much better inhibitor of Fli-1 than A661; A665 has a predicted binding energy of $-8.51 \mathrm{KJ} / \mathrm{Mol}$ compared to A661 with $-7.53 \mathrm{KJ} / \mathrm{Mol}$ (Fig. 3a). Consistent with this analysis, western blot analysis revealed that Fli-1 expression was more downregulated by A665 than by A661 (Fig. 3b). MTM showed hydrogen-bond interactions with 4 nucleotides: DC5, DG6, DG7 and DC6. A661 in addition to hydrophobic interactions with nucleotide DC6 also formed hydrogen-bond interaction with $\mathrm{H}_{2} \mathrm{O}$ and coordination bonds with $\mathrm{Zn}^{2+}$ ions (Fig. 3a). A665 formed hydrophobic interaction with nucleotides DG6 and DG7, hydrogen-bond interaction with $\mathrm{H}_{2} \mathrm{O}$ and coordination bonds with $\mathrm{Zn}^{2+}$ ions. Thus, A661/A665 may represent novel anti-Fli-1 compounds with unique interaction with the DNA-binding sites of Fli-1. 


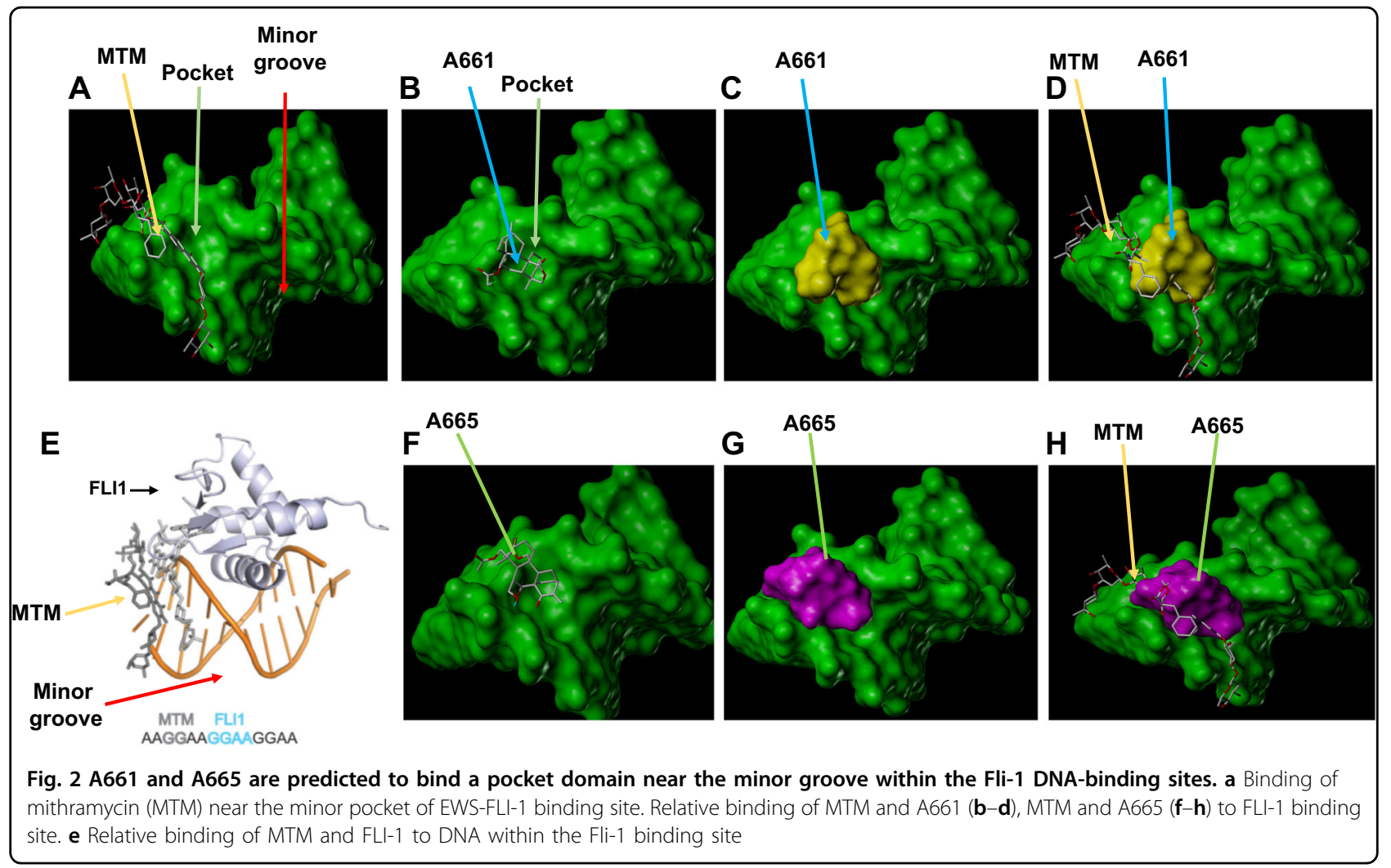

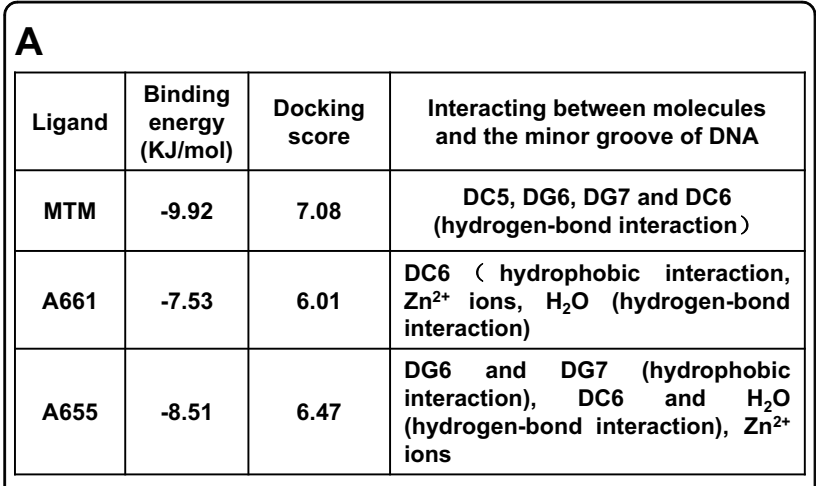

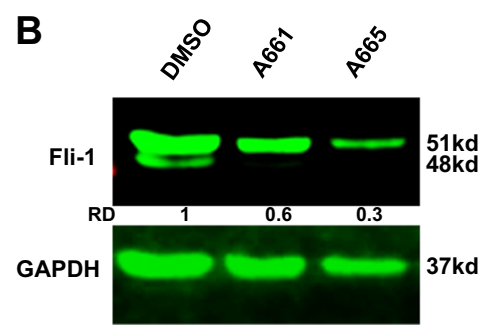

Fig. 3 High affinity of A661 and A665 compounds binding to the Fli-1 consensus DNA-binding sites. a Properties of A661 and A665 binding affinities to the Fli-1 binding sites. $\mathbf{b}$ Western blot of HEL cells treated with $2.5 \mu \mathrm{M}$ of A661 or A665. GAPDH was used as loading control
Post-transcriptional regulation of Fli- 1 by miR-145

The observed downregulation of Fli-1 protein expression by A661 and A665 (Fig. 1f, g) was not at the transcriptional level (Supplemental Fig. 1). The proteasome inhibitor MG132 did not rescue Fli-1 expression indicating that its reduced expression was not due to protein degradation (Supplemental Fig. 5). Previous studies demonstrated that microRNA-145 (miR-145) controls Fli1 protein translation ${ }^{26-29}$. Remarkably, we found that treatment with A661 and A665 resulted in a significant upregulation of miR145 expression, with A665 having a stronger effect (Fig. 4a). To validate the role of miR145 in drug-induced inhibition of Fli-1, we transduced HEL cells with a pre-miR145 lentivirus or control vectors, sorted GFP-positive cells by flow cytometry and photographed the cells two days post selection with puromycin (Fig. 4b). Significantly lower viability was seen with the pre-miR145 transduced cells compared to vector control (Fig. 4b). Furthermore, miR145 overexpression (Fig. 4c) significantly suppressed Fli-1 protein level (Fig. 4d) and reduced cell survival (Fig. 4b, e).

To complement these results, we knocked down endogenous miR145 by transducing GFP tagged lenti-shmiR145 or scrambled control vector into HEL cells. GFP positive sh-miR145 and scrambled control expressing 


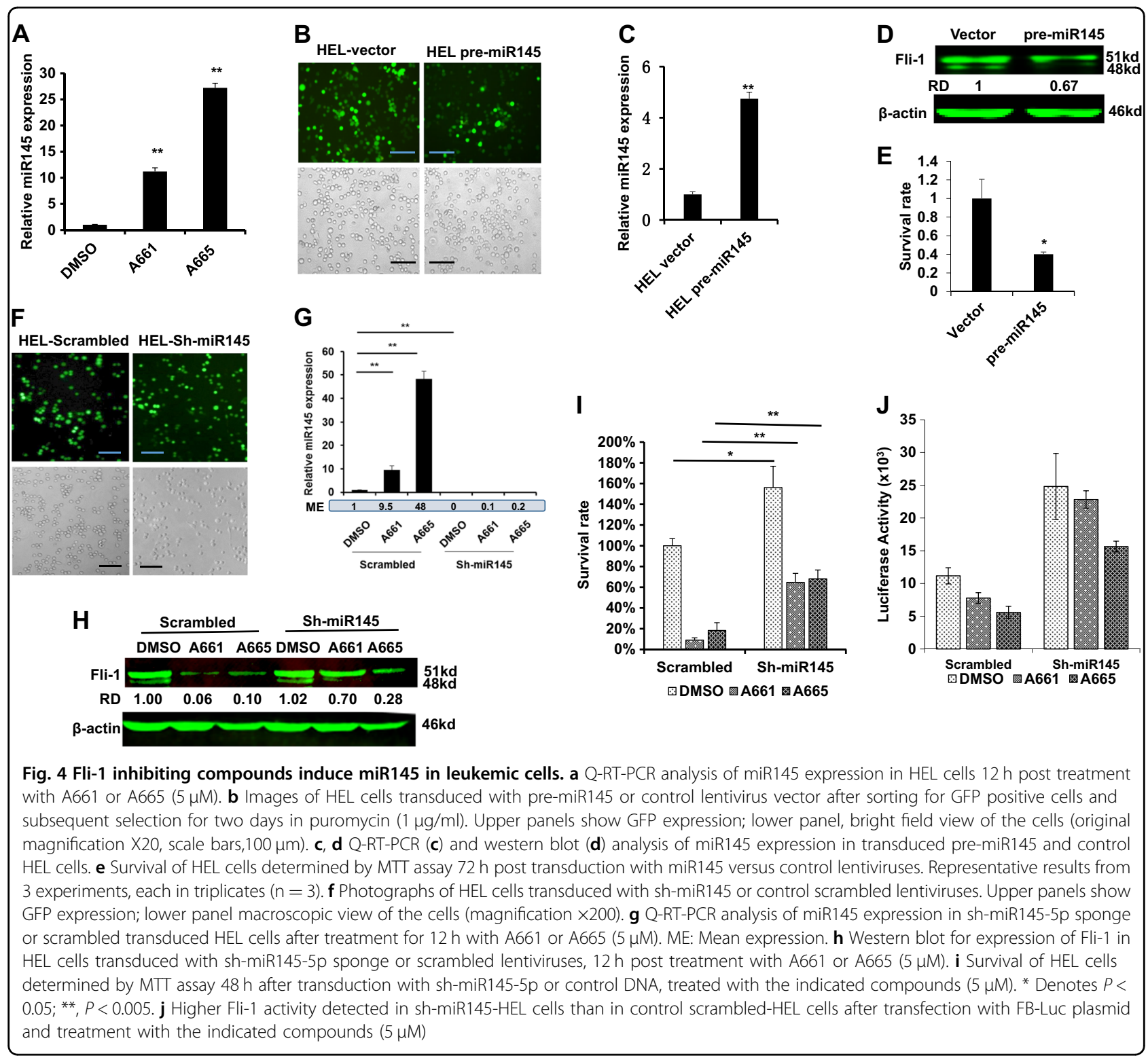

cells exhibited similar cell number (Fig. 4f). Expression of miR-145, which was induced by A661 or A665, was completely diminished by sh-miR145 expression (Fig. 4g). Moreover, expression of the Fli-1 protein, which was suppressed by A661 or A665, was rescued by sh-miR145 (Fig. 4h). In accordance, treatment of sh-miR145 expressing cells with A661 or A665 resulted in a higher survival rate (Fig. 4i) and increased Fli-1 reporter activity following transient transfection with the FB-Luc gene (Fig. 4j). Moreover, miR-145 expression was not induced in Fli-1 negative K562 cells (Supplemental Fig. 6), demonstrating that A661 and A665 induce miR145 via Fli-1, and thus suppress the growth of leukemic cells at least in part through a Fli-1-miR145 auto-inhibition loop (see below).

\section{Fli-1 negatively regulates miR-145 transcription}

The aforementioned results suggested that Fli-1 negatively regulates the miR-145 promoter as had previously been reported for EWS-Fli- $1^{29}$. We tested this possibility using a miR-145 promoter reporter plasmid, miR145-Luc. Overexpression of Fli-1 significantly suppressed the miR145 promoter in this assay (Fig. 5a). Conversely, knockdown of Fli-1 via shRNA significantly increased endogenous expression of miR-145 (Fig. 5b-d).

The human miR145 promoter contains 3 ETS-binding sites (FBS1-FBS3; Fig. 5e). Chromatin immunoprecipitation (ChIP) analysis confirmed binding of FLI1 to a region surrounding FBS1, but not FBS2-3 (Fig. 5f, g). As a positive control, antibody for RNA polymerase II immunoprecipitated a fragment of the miR-145 promoter 


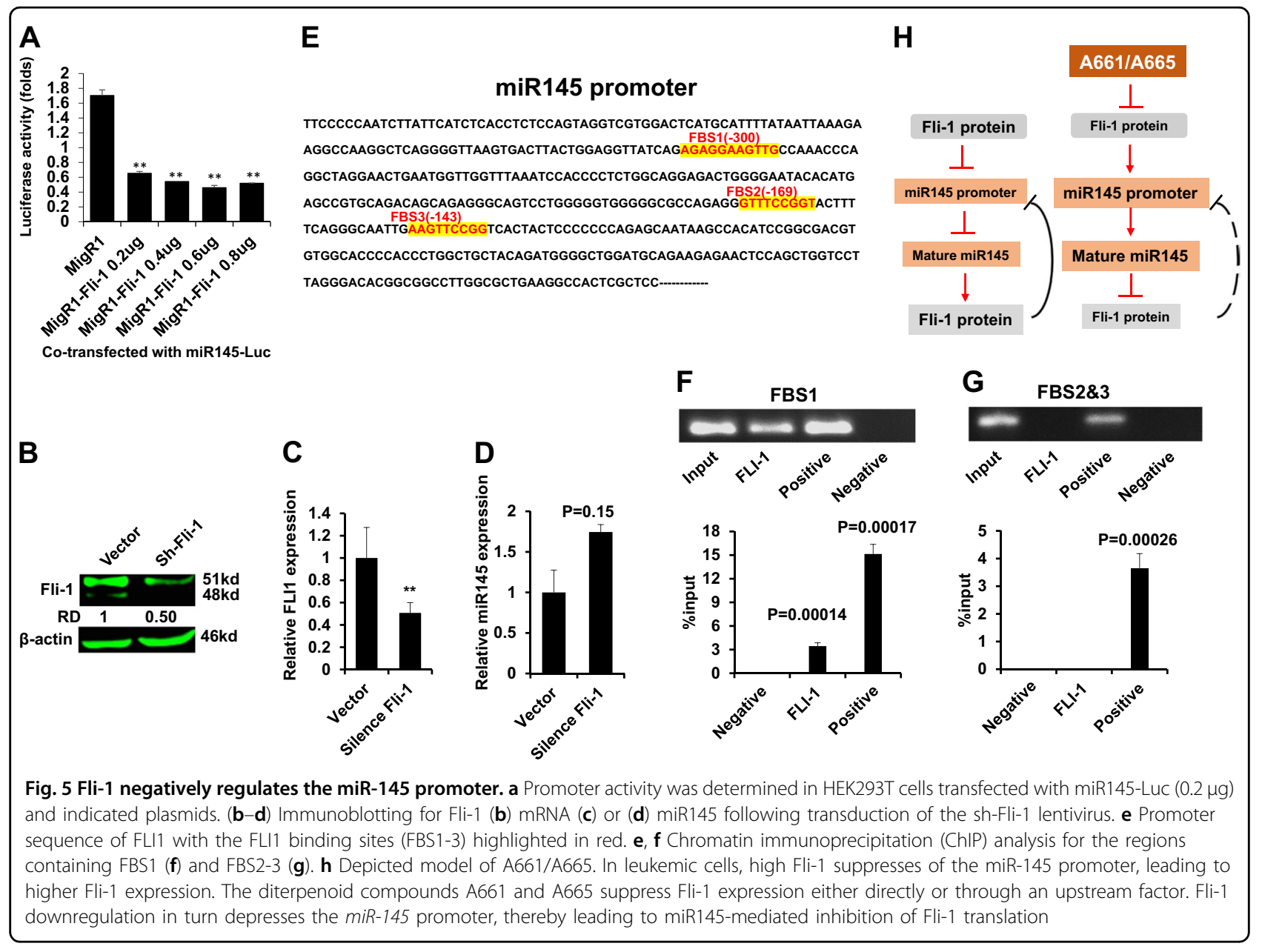

containing all three sites. Together, these results suggest a model in which in leukemic cells, high Fli-1 expression suppresses miR145 transcription, leading to enhanced Fli1 translation that further suppresses miR145 (Fig. 5h left). Upon drug treatment, Fli-1 inhibition induces miR-145 that creates the opposite effect, leading to a negative feedback loop that extinguishes Fli-1 expression (Fig. 5h right).

Fli-1 inhibition via A661/A665 or shRNA suppresses growth of leukemic cells in culture and in a preclinical model of leukemia

Fli-1 expression is elevated in B-cell and myeloid tumors $^{7,30}$. Both A661 and A665 significantly reduced Fli1 protein expression in the B-cell lymphoma cells line Daudi (Fig. 6a). Fli-1 inhibition by these drugs was associated with a dramatic upregulation of miR-145 (Fig. 6b) and reduced survival in culture (Fig. 1e). A drastic decrease in Fli-1 expression was also detected in B-chronic lymphocytic leukemia (B-CLL) cells isolated from 7 independent patients, $12 \mathrm{~h}$ post incubation with A661 or A665 (Fig. 7a). Twenty-four-hour treatment with
A661/A665 led to a significant reduction in cell viability (Fig. 7b), demonstrating the utility of these compounds for the treatment of B-cell lymphoma.

To investigate the ability of A661 and A661 to inhibit leukemogenesis in vivo, we used an animal model of Friend-Murine leukemia virus (F-MuLV)-induced erythroleukemia, driven by Fli- ${ }^{10}$. In this model, F-MuLV injection into newborn Balb/c mice induces transformation 4-6 weeks post-infection ${ }^{31}$. Mice were then treated every other day with A661 or A665 (3 mg/ $\mathrm{kg}$ ) for a period of 12 days. Treatment of leukemic mice $(n=7)$ with A661 or to a lesser extent with A665 for this short period resulted in significant inhibition of leukemogenesis ${ }^{10,16}$. While the size of spleens was similar in control versus drug-treated cells, mice treated with A661/A665 were less anemic, an indication of tumor inhibition (Fig. 6d, e). Thus, A661 and A665 exhibit anti-leukemic activity against leukemias driven by Fli-1 overexpression.

\section{Discussion}

Fli-1 is overexpressed in various cancers and diseases rendering it an important target for drug development ${ }^{7}$. 

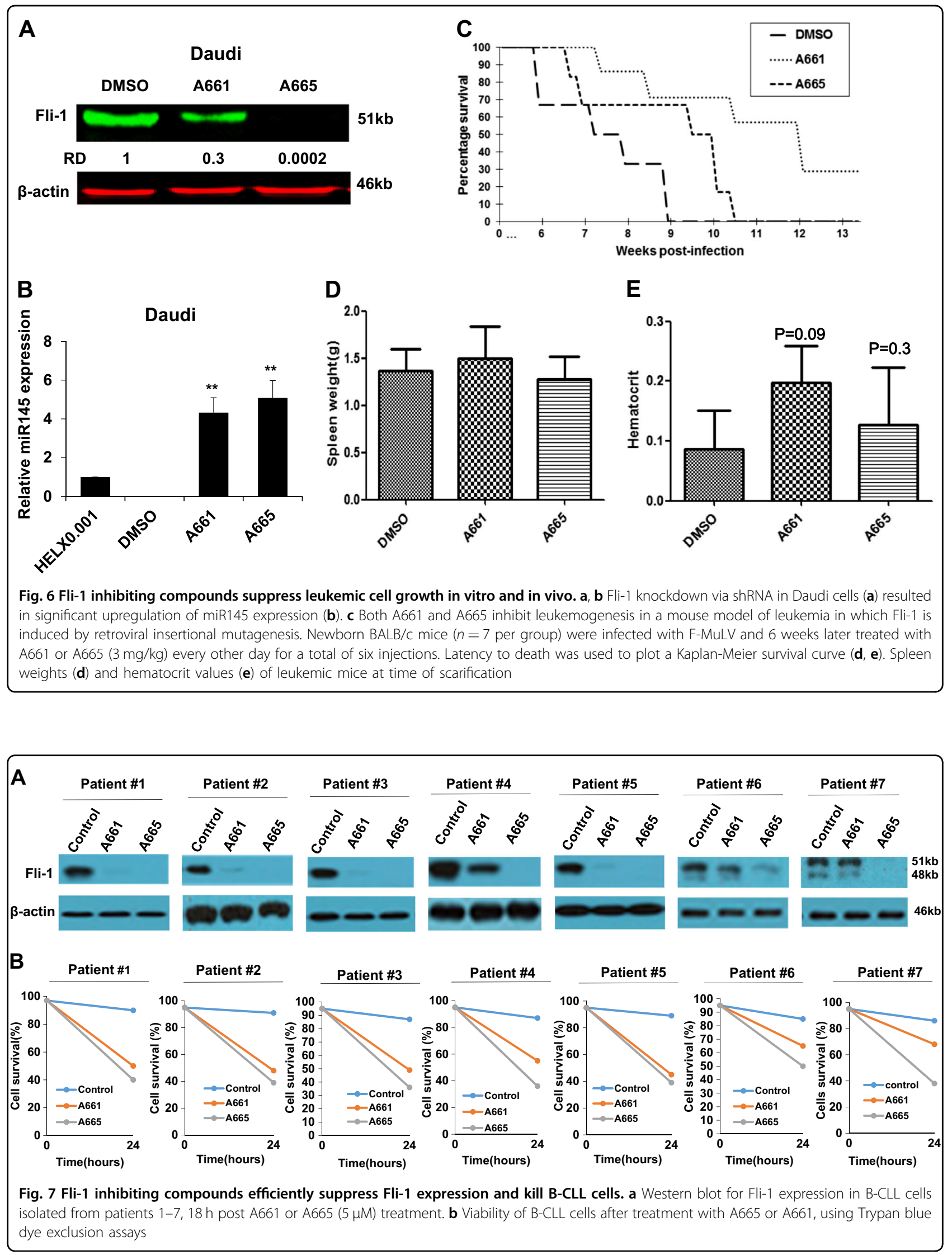
By screening a library of compounds isolated from medicinal plants in China, we now identified two structurally related diterpenoids, A661 and A665, with potent anti-Fli1 activity. These inhibitors downregulate Fli- 1 protein and further extinguish its expression post-transcriptionally as miR-145, a suppressor of Fli-1 protein translation, is upregulated upon drug-mediated inhibition of Fli-1. A661 and A665 induced differentiation and apoptosis and attenuated the growth of B-cell lymphomas expressing high levels of Fli-1 in vitro, and erythroleukemia in a preclinical model in vivo. Thus, these drugs exert very similar effects as shRNA-mediated knockdown of Fli-1. The ability of A661 and A665 to block growth of B-cell lymphomas from multiple independent patients as well as leukemogenesis in a preclinical model point to their potential application as drugs for the treatment of leukemias and other cancers driven by Fli-1 overexpression.

The anti-Fli-1 diterpenoids displayed high affinity to the DNA-binding sites of FLI1, a region that was previously reported to be targeted by Mithramycin, a potent inhibitor of the EWS-FLI1 fusion oncoprotein ${ }^{25}$. This binding inhibits Fli-1 function leading to transcriptional modulation of downstream target genes involved in cell growth, differentiation, and apoptosis. Among these targets is miR-145, which was previously reported to be regulated by EWS-Fli- ${ }^{26-29}$. MiR-145 targets Fli-1 as well as other genes that play critical roles in progression of various cancers including those of the prostate, breast and colon $^{32-35}$. Our study demonstrates that Fli-1 inhibition by the diterpenoid compounds upregulates miR-145 expression, leading to downregulation of Fli-1 and consequently to inhibition of leukemia. High miR145 expression induced by low Fli-1 likely further suppresses growth by targeting other genes such as the RAS pathway, TGFBR2 and others ${ }^{32-37}$. ShRNA-mediated knockdown of miR-145 in drug-treated leukemias only partially rescued survival, indicating the importance of other Fli-1 targets during leukemogenesis. Thus, Fli-1 inhibition blocks tumor growth in part by upregulating miR145, which is kept in check in untreated tumors by high levels of Fli-1. How do A661 and A665 inhibit Fli-1? The simplest model is that these compounds, like mithramycin ${ }^{25}$, directly interact with the Fli-1 DNA-binding sites to inhibit its function. However, we cannot rule out the possibility that A661 and A665 inhibit Fli-1 indirectly either by blocking an upstream regulator. Future analysis is required to confirm a direct binding of A661 and A665 to Fli-1 or identify new upstream targets. However, we showed that these drugs in part set in motion a Fli1-miR-145 autoregulatory loop that extinguishes Fli-1 expression and suppresses leukemia.

Our study confirms the negative regulation of the miR145 promoter by Fli-1, and further shows that it is mediated by the FBS1 element in the miR145 promoter.
We show that inhibition of Fli-1 by A661/A665 upregulated Fli-1 transcription. This result suggests that Fli-1 may negatively regulate itself, likely through ETS binding sites in its promoter ${ }^{17,18}$.

We demonstrated that A661 and A665 inhibit proliferation not only of cancer cells expressing Fli-1, but also of cancer cells lacking Fli-1 expression. This antiproliferative activity is mediated through other target(s) in this context. Indeed, a similar diterpenoid derivative, 15-oxospiramilactone, was previously reported to block the WNT signaling in breast cancer cells ${ }^{15}$, and this finding has been confirmed in our study (unpublished data). Our compounds in part by suppressing Fli-1 and the WNT pathways may cooperatively exert better inhibitory response than each target alone. Thus, A661 and A665 may offer an excellent new treatment for tumors, such as triple-negative breast cancer ${ }^{37}$, in which both Fli-1 and MYC drive cell survival and proliferation.

In summary, two anti-Fli-1 diterpenoid compounds identified in this study, A661 and A665, induce cell cycle arrest and apoptosis of leukemic cells in culture and inhibit leukemogenesis in vivo. These inhibitors disrupt an autoregulatory loop that maintains high level of Fli-1 via Fli-1-induced suppression of its negative regulator, miR145. These diterpenoids may provide important new tools for the treatment of leukemia and lymphomas driven by overexpression of Fli-1.

\section{Materials and methods \\ Cell lines}

Mycoplasma negative cell lines originated from erythroleukemia (CB7 and HEL), human embryonic kidney (HEK293T), B-cell lymphoma (Daudi) were maintained in Dulbecco's Modified Eagle Medium supplemented with $5 \%$ fetal bovine serum (HyClone, GE Healthcare, Australia). Human B-CLL cells were isolated and grown, as described previously ${ }^{38}$.

\section{Leukemia induction and in vivo compound treatment}

Newborn BALB/c mice were inoculated intraperitoneally (i.p.) by F-MuLV as described ${ }^{16}$. Six weeks post viral infection, mice were injected i.p., every other day for a total of six inoculations with A661 or A665 compounds ( $3 \mathrm{mg} / \mathrm{kg}$ body weight) or control DMSO and monitored for development of severe leukemia. Mice showing signs of late-stage disease were sacrificed and \% survival was calculated as described ${ }^{16}$.

\section{Cell cycle and apoptosis}

Apoptosis and cell cycle analysis were described elsewhere $^{16}$. In brief, cells were incubated with compounds or DMSO for $48 \mathrm{~h}$ and cells then washed by cold PBS. For apoptosis experiment, cells were stained by Annexin V and PI apoptosis detection Kit (BD Biosciences, Franklin 
Lakes, NJ) following the kit guidelines and analyzed by flow cytometer. For cell cycle analysis, cells were fixed by cold $75 \%$ ethanol overnight at $-20^{\circ} \mathrm{C}$, washed by cold PBS, stained in PI for $40 \mathrm{~min}$ at $37^{\circ} \mathrm{C}$, then analyzed by flow cytometer.

\section{Compound screening, luciferase assay, and promoter analysis}

A collection of 2000 compounds, isolated from Chinese medicinal plants, was used to screen for anti-Fli-1 activity, as described ${ }^{16}$. In brief, HEK293T cells were cotransfected with FB-Luc $(1.25 \mu \mathrm{g})$ and MigR1 $(1.25 \mu \mathrm{g})$ or MigR1-Fli-1 (1.25 $\mu \mathrm{g})$ expression vector for $24 \mathrm{~h}$ using Lipofectamine 2000 (Life Technology, Beijing, China) following the manufacturer's protocol. In these transfection experiments, renilla luciferase was used as internal control to determine transfection efficiency, according to manufacturer recommendations (Promega, Fitchburg, USA). The transfected cells were plated into 96 well plates, incubated for $12 \mathrm{~h}$ and then treated with compounds $(5 \mu \mathrm{M})$ or other indicated compounds for additional $12 \mathrm{~h}$. Luciferase activity was determined, as described $^{9}$. MiR145 promoter fused to luciferase gene (miR145-Luc) was obtained from Kenneth Kosik (addgene, Cambridge, MA, USA, plasmid \# 21500) ${ }^{39}$.

\section{Drug studies}

Triplicates of cancer cell lines $\left(1 \times 10^{4}\right)$ were plated in 96 well plates and treated with various concentration of compounds for 3 days. Cells were then subjected to Tetrazolium dye (MTT) assay, as described ${ }^{16}$. The IC50 (the concentration of compound required to reduce $50 \%$ of cell viability) was calculated based on reading between drug and DMSO treated cells.

\section{Western blotting, immunoprecipitation, and inhibitory compounds}

Methods for Western blotting was described elsewhere $^{16}$. Polyclonal rabbit antibody for Fli-1 were obtained from Abcam (Abcam, Cambridge, UK); ERK and phosphoERK from Cell Signalling Technology (CST, Danvers, MA01923), $\beta$-actin, $\beta$-Tubulin, BCL-2 from ProtoTechnology (Protein-Tech, Bucuresti, Romania) and goat-anti-mouse, and goat anti-rabbit HRP-conjugated (Promega). Antibody dilution was according to the manufacturer instructions. Imaging of proteins was performed using Oddessy system (Li-Cor biosciences, Lincoln, USA). In experiments described in Fig. 7a, we used ECL detection system for western blotting, as described ${ }^{9}$.

\section{Total RNA and microRNA isolation and quantitative real- time PCR}

Total RNA was isolated from cell lines with TRIzol (Invitrogen Life Technologies, Carlsbad, USA) according to the manufacturer's protocol. A NanoDrop 2000 spectrophotometer (Thermo Scientific, USA) was used to measure RNA concentration. Reverse transcription reactions were performed with PrimeScript RT Reagent kit (Takara, Dalian, China), and quantitative real-time PCR (qRT-PCR) was performed using FastStart Universal SYBR Green Master (Roche, Mannheim, Germany) and the Step One Plus Real-time PCR system (Applied Biosystems, Singapore, Singapore). Expression was normalized to $\beta$-actin level. Primer sequences were as followed in Supplemental Table I. For miR-145 detection, miRNA isolated from cell lines using miRNA extract kit (Haigene, Haerbing, China), according to the manufacturer's protocol. Reverse transcription reactions were performed with RevertAid First Strand cDNA Synthesis Kit (Thermo scientific, Lithuania, EU). miRNA-specific primers were purchased from RiboBio (RiboBio, Guangzhou, China), and the relative miR-145 expression level was normalized to U6. The 2- $\Delta \Delta \mathrm{Ct}$ method was used for relative quantification. All experiments were performed in biological triplicates, each in triplicates $(n=3)$.

\section{Plasmid construction, lentiviral production, and infection}

The pre-miR-145 expression construct was generated by cloning pre-miR-145 sequence (Supplemental Table II) into the AsiSI-MluI sites of pLent-puro-GFP (Vigenebio, Shandong, China). A Fli-1 shRNA expression construct was generated by cloning Fli-1 4in1shRNA sequence (Supplemental Table II) into the BbsI-BcuI sites of the PHS-4in1shRNA-GFP lentivirus vector (Vigenebio). For lentivirus production, packaging plasmids psPAX2 and PMD2.D (a gift from Didier Tronon, Addgene plasmid \# 12259 and 12260) and pLent-puro-GFP-mir145 or Fli-1 PSH-4in1shRNA-GFP were co-transfected into HEK293T cells, as described ${ }^{16}$. $48 \mathrm{~h}$ post transfection, supernatants were harvested, viruses were centrifuged at $2500 \times g$ for $10 \mathrm{~min}$ and filtered through $0.45 \mu \mathrm{m}$ filters. For infection, HEL cells were cultured and grown in the presence of fresh supernatant of lentivirus producing cells. After $24 \mathrm{~h}$ infection, medium was changed, and cells either sorted for GFP positive cells and/or were grown in presence of puromycin (Solanbio, Beijing, China), until -resistant cells were obtained.

The Sh-miR145 expression construct (Has-miR-145-5p) was generated by cloning Sh-miR145 sequence (Supplemental Table I) into the SgfI-MluI sites of PAV-CAGMIR plasmid (Vigenebio). Has-miR-145-5p sponge or PAV-CAG-MIR vector was transfected into HEL cells with ViaFect Transfection Reagent (Promega, Madison, USA), following the manufacturer's protocol. GFP- positive cells were sorted using fluorescence-activated cell sorting (FACS) 2 days post-transduction. After 10 days in culture, cells were sorted again for selection of high GFP expressing cells. 


\section{Chromatin immunoprecipitation (ChIP) and quantitative PCR}

HEL cells $\left(1 \times 10^{6}\right)$ were washed and crosslinked as previously described ${ }^{10}$. Fixed cells were washed and resuspended in lysis buffer Magna Chip G kits (Millipore, MA, USA) and sonicated using the sonics vibra VCX150 (Scientz biotechnology, Ningbo, China). A fragmented chromatin aliquot was removed for input control. Protein $\mathrm{G}$ sepharose beads were added and incubated for $1 \mathrm{~h}$. Immunoprecipitations were performed overnight at $4{ }^{\circ} \mathrm{C}$ with $1 \mu \mathrm{g}$ of Fli-1 (Abcam), $1 \mu \mathrm{g}$ of anti-RNA Polymerase II (Millipore/MERK, Darmstadt, Germany) or nonspecific normal rabbit immunoglobulin G (IgG; Abcam) antibodies. Precipitates were washed, and reverse crosslinking followed instruction for Magna Chip G kits (Millipore). DNA was incubated with proteinase $\mathrm{K}$ at $50^{\circ} \mathrm{C}$ for $2 \mathrm{~h}$, purified with phenol chloroform and resuspended in TE buffer. Real-time PCR was performed to amplify two mir145 promoter regions containing the Fli-1 binding site 1 FBS1(position -482 to -205 , primers in Supplemental Table II) and FBS2/FBS-3 (position -231 to +19 ). The percentage of input was calculated by Q-RT-PCR based on the intensity of amplified $f l i-1$ DNA divided by the amplified input DNA.

\section{Animal survival experiments and statistical analysis}

Mice survival rates were computed and plotted according to the nonparametric Kaplan-Meier analysis. Statistical analysis was performed using the two-tailed Student $t$-test with significance considered at $P<0.05$, and by analysis of variance using Origin 3.5 software (Microcal Software, Northampton, MA, USA).

\section{Computation docking analysis}

The 3-dimensional structures of A661 and A665 were analyzed and drawn in chen sketch. The protein Crystallographic structure of FLI-1 (PDB ID: 5JVT) was obtained from www.rcsb.org. Auto Dock tools 1.5.6. were used to compute molecular docking simulations, as described $^{40}$.

\section{Animal care}

Animal care was in accordance with guidelines of the Guizhou Medical University and China Council of Animal Care.

\section{Acknowledgements}

This work was supported by research grants from the Science and Technology Department of Guizhou Province innovation and project grants $(6012,4001)$ the Thousand Talent Program of China (WQ20135200171) and The Natural National Science Foundation of China (81472609) to YBD.

\section{Author details}

'State Key Laboratory for Functions and Applications of Medicinal Plants, Guizhou Medical University, Guiyang 550025, China. ${ }^{2}$ The Key Laboratory of Chemistry for Natural Products of Guizhou Province and Chinese Academic of
Sciences, Guiyang, Guizhou 550014, China. ${ }^{3}$ State Key Laboratory of Phytochemistry and Plant Resources in West China, Kunming Institute of Botany, Chinese Academy of Sciences, Kunming, China. ${ }^{4}$ Department of Anatomy, Norman Bethune College of Medicine, Jilin University, Changchun, China. ${ }^{5}$ Biology Platform, Sunnybrook Research Institute, Toronto, Canada. ${ }^{6}$ Department of Medicine, University of Toronto, Toronto, Ontario, Canada. ${ }^{7}$ Division of Advanced Diagnostics, Toronto General Research InstituteUniversity Health Network, Toronto, Ontario, Canada

Conflict of interest

The authors declare that they have no conflict of interest.

\section{Publisher's note}

Springer Nature remains neutral with regard to jurisdictional claims in published maps and institutional affiliations.

Supplementary Information accompanies this paper at (https://doi.org/ 10.1038/s41419-019-1363-1).

Received: 16 August 2018 Revised: 9 January 2019 Accepted: 9 January 2019

Published online: 11 February 2019

\section{References}

1. Whiteside, T. L. The tumor microenvironment and its role in promoting tumor growth. Oncogene 27, 5904-5912 (2008).

2. Ben-David, Y. \& Bernstein, A. Friend virus-induced erythroleukemia and the multistage nature of cancer. Cell 66, 831-834 (1991).

3. Ben-David, Y., Giddens, E. B. \& Bernstein, A. Identification of a common proviral integration site in erythroleukemic cells induced by Friend Murine Leukemia Virus (F-MuLV). Proc. Natl Acad. Sci. USA 87, 1332-1336 (1990).

4. Ben-David, Y., Giddens, E. B., Letwin, K. \& Bernstein, A. Erythroleukemia induction by Friend murine leukemia virus: insertional activation of a new member of the ets gene family, Fli-1, closely linked to c-ets-1. Genes Dev. 5 908-918 (1991).

5. Tamir, A. et al. Fli-1, an Ets-related transcription factor, regulates erythropoietininduced erythroid proliferation and differentiation: evidence for direct transcriptional repression of the Rb gene during differentiation. Mol. Cell Biol. 19, 4452-4464 (1999).

6. Delattre, O. et al. Gene fusion with an ETS DNA-binding domain caused by chromosome translocation in human tumours. Nature 359, 162-165 (1992).

7. Li, Y., Luo, H., Liu, T., Zacksenhaus, E. \& Ben-David, Y. The ets transcription factor Fli-1 in development, cancer and disease. Oncogene 34, 2022-2031 (2015).

8. Lennard, R. M. L., Nowling, T. K., Brandon, D., Watson, D. K. \& Zhang, X. K. Fli-1 controls transcription from the MCP-1 gene promoter, which may provide a novel mechanism for chemokine and cytokine activation. Mol. Immunol. 63, 566-573 (2015).

9. Cui, J. W., Vecchiarelli-Federico, L. M., Li, Y. J., Wang, G. J. \& Ben-David, Y. Continuous Fli-1 expression plays an essential role in the proliferation and survival of F-MuLV-induced erythroleukemia and human erythroleukemia. Leukemia 23, 1311-1319 (2009).

10. Li, Y. J. et al. Drug-mediated inhibition of Fli-1 for the treatment of leukemia. Blood Cancer J. 2, e54 (2012).

11. Grohar, P. J. et al. Identification of an inhibitor of the EWS-FLI1 oncogenic transcription factor by high-throughput screening. J. Natl. Cancer Inst. 103, 962-978 (2011).

12. Erkizan, H. V. et al. A small molecule blocking oncogenic protein EWS-FLI1 interaction with RNA helicase A inhibits growth of Ewing's sarcoma. Nat. Med 15, 750-756 (2009).

13. Grohar, P. J. et al. Ecteinascidin 743 interferes with the activity of EWS-FLI1 in Ewing sarcoma cells. Neoplasia 13, 145-153 (2011).

14. Boro, A. et al. Small-molecule screen identifies modulators of EWS/FLI1 target gene expression and cell survival in Ewing's sarcoma. Int. J. Cancer 131, 2153-2164 (2012)

15. Wang, W., Liu, H., Wang, S., Hao, X. \& Li, L. A diterpenoid derivative 15oxospiramilactone inhibits Wnt/beta-catenin signaling and colon cancer cell tumorigenesis. Cell Res. 21, 730-740 (2011). 
16. Liu, T. et al. A screen for Fli-1 transcriptional modulators identifies PKC agonists that induce erythroid to megakaryocytic differentiation and suppress leukemogenesis. Oncotarget 8, 16728-16743 (2017).

17. Barbeau, B., Barat, C., Bergeron, D. \& Rassart, E. The GATA-1 and Spi-1 transcriptional factors bind to a GATAVEBS dual element in the Fli-1 exon 1. Oncogene 18, 5535-5545 (1999).

18. Barbeau, B., Bergeron, D., Beaulieu, M., Nadjem, Z. \& Rassart, E. Characterization of the human and mouse Fli-1 promoter regions. Biochim. Biophys. Acta 1307, 220-232 (1996)

19. Lakhanpal, G. K. et al. The inositol phosphatase SHIP-1 is negatively regulated by Fli-1 and its loss accelerates leukemogenesis. Blood 116, 428-436 (2010).

20. Kanaji, S. et al. Thrombopoietin initiates demethylation-based transcription of GP6 during megakaryocyte differentiation. Blood 105, 3888-3892 (2005).

21. Moussa, O. et al. Thrombocytopenia in mice lacking the carboxy-terminal regulatory domain of the Ets transcription factor Fli1. Mol. Cell Biol. 30, 5194-5206 (2010).

22. Athanasiou, M., Mavrothalassitis, G., Sun-Hoffman, L. \& Blair, D. G. FLI-1 is a suppressor of erythroid differentiation in human hematopoietic cells. Leukemia 14, 439-445 (2000).

23. Vecchiarelli-Federico, L. M. et al. Fli-1 overexpression in erythroleukemic cells promotes erythroid de-differentiation while Spi-1/PU.1 exerts the opposite effect. Int J. Oncol. 51, 456-466 (2017).

24. Anantharaman, A. et al. Role of helix-loop-helix proteins during differentiation of erythroid cells. Mol. Cell Biol. 31, 1332-1343 (2011).

25. Hou, C. et al. Structures of mithramycin analogues bound to DNA and implications for targeting transcription factor FLI1. Nucleic Acids Res. 44, 8990-9004 (2016)

26. Wu, P. et al. miR-145 promotes osteosarcoma growth by reducing expression of the transcription factor friend leukemia virus integration 1. Oncotarget 7 , 42241-42251 (2016).

27. Zhang, J. et al. Putative tumor suppressor miR-145 inhibits colon cancer cell growth by targeting oncogene Friend leukemia virus integration 1 gene. Cancer 117, 86-95 (2011).
28. Larsson, E. et al. Discovery of microvascular miRNAs using public gene expression data: miR-145 is expressed in pericytes and is a regulator of Fli1. Genome Med 1, 108 (2009).

29. Ban, J. et al. Hsa-mir-145 is the top EWS-FLI1-repressed microRNA involved in a positive feedback loop in Ewing's sarcoma. Oncogene 30, 2173-2180 (2011).

30. Bradshaw, S., Zheng, W. J., Tsoi, L. C., Gilkeson, G. \& Zhang, X. K. A role for Fli-1 in B cell proliferation: implications for SLE pathogenesis. Clin. Immunol. 129, 19-30 (2008).

31. Howard, J. C., Yousefi, S., Cheong, G., Bernstein, A. \& Ben-David, Y. Temporal order and functional analysis of mutations within the Fli-1 and p53 genes during the erythroleukemias induced by F-MuLV. Oncogene 8, 2721-2729 (1993).

32. Ma, Y. et al. Candidate microRNA biomarkers in human colorectal cancer: systematic review profiling studies and experimental validation. Int J. Cancer 130, 2077-2087 (2012).

33. Zhang, $X . \& W u, J$. Prognostic role of microRNA-145 in prostate cancer: $A$ systems review and meta-analysis. Prostate Int 3, 71-74 (2015).

34. Teoh, S. L. \& Das, S. The Role of MicroRNAs in Diagnosis, Prognosis, Metastasis and Resistant Cases in Breast Cancer. Curr. Pharm. Des. 23, 1845-1859 (2017).

35. Wang, S. et al. microRNA-143/145 loss induces Ras signaling to promote aggressive Pten-deficient basal-like breast cancer. JCI Insight 2, e93313 (2017)

36. Zhao, $\mathrm{N}$. et al. MicroRNA miR145 regulates TGFBR2 expression and matrix synthesis in vascular smooth muscle cells. Circ. Res. 116, 23-34 (2015).

37. Sakurai, $\mathrm{T}$. et al. Functional roles of Fli-1, a member of the Ets family of transcription factors, in human breast malignancy. Cancer Sci. 98, 1775-1784 (2007).

38. Li, Y. et al. The miR-17-92 cluster expands multipotent hematopoietic progenitors whereas imbalanced expression of its individual oncogenic miRNAs promotes leukemia in mice. Blood 119, 4486-4498 (2012).

39. Xu, N., Papagiannakopoulos, T., Pan, G., Thomson, J. A. \& Kosik, K. S. MicroRNA145 regulates OCT4, SOX2, and KLF4 and represses pluripotency in human embryonic stem cells. Cell 137, 647-658 (2009).

40. Morris, G. M. et al. Auto-Dock 4 and AutoDockTools 4: automated docking with selective receptor flexibility. J. Comput. Chem. 30, 2785-2791 (2009). 